\title{
The Testing and Visualization of the Singularities of the Mutual Intersection of a Tetrahedron and a Quadric (Chasles' Theorem)
}

\author{
A. L. Kheyfets ${ }^{1}$ \\ South Ural State University, Chelyabinsk, Russia \\ 1. ORCID: 0000-0001-6490-359X, heifets@yandex.ru
}

\begin{abstract}
This article presents the results of the experimental research and testing of Chasles' historical theorem. The theorem shows the singularities of the intersection of an arbitrary tetrahedron and an arbitrary quadric (second-order surface). The need for testing is preconditioned by the absence of a proof of the theorem and the complexity of its perception in Chasles' version.

The experiments included the construction, visualization, and study of $3 \mathrm{D}$ computer models using AutoCAD and SolidWorks. All forms of quadrics are considered in their different relative positions to the tetrahedron. The experimental procedure is considered in detail and the accuracy of the results is estimated. The author tested all the intersection variants given in the theorem: the edges intersect a quadric, the vertices belong to a quadric, the edges are tangent to a quadric, the faces are tangent to a quadric, etc. The experiments confirmed the scientific novelty of the theorem, which is that four intersecting straight lines drawn according to the algorithm of the theorem belong to the surface a single one-sheeted hyperboloid.

The paper investigated in detail the form of the theorem when the planes drawn through the edges of a tetrahedron are tangent and enclose the quadric. It shows that there are 4,096 combinations of plane positions. Only 64 of these combinations, obtained using AutoLisp, lead to the realization of the theorem. This conclusion supplements the theorem.

The results differ from the theorem in two forms. The paper presents a proof of one of the theorem forms, although a universal proof of the theorem has yet to be developed. The models and algorithms can be used when teaching computer geometric simulation.

Keywords: 3D computer geometrical simulation, Michel Chasles, tetrahedron, quadrics, Pascal's hexagon, Auto-CAD, AutoLisp.
\end{abstract}

\section{Introduction}

Polyhedrons and quadrics (second-order surfaces) are widespread geometric elements in architectural design. The shape and interaction of these elements have been studied and investigated in detail over the centuries-long history of the development of geometry. Nevertheless, in [1], we find a theorem revealing one more side of this interaction. This is Chasles' theorem, which shows some previously unexamined singularities of the intersection of polyhedrons with quadrics.

Let us present the formulation of Chasles' theorem given in [2]:

"The planes determined by the points of intersection of some second-order surfaces with three edges of the tetrahedron converging at one vertex intersect the opposite faces along the straight lines, which are rectilinear generators of one hyperboloid."[author's translator]

This theorem has scientific novelty as it is known that a one-sheeted hyperboloid (Hyper) is determined by three skew straight lines [3-6], which are taken as its guides. As a result 
of applying the algorithm of Chasles' theorem, we obtain four segments belonging to the family of guides of a single Hyper. That is, if you construct a Hyper by three segments arbitrarily selected from these four, the remaining segment will also belong to the surface of this Hyper. This is a previously unknown relationship between the intersection of a tetrahedron with a quadric and a one-sheeted hyperboloid.

Chasles did not prove his theorem, but showed its numerous manifestations. Chasles drew an analogy between his theorem and Pascal's theorem [7, 8], which was not proved by its author and therefore aroused a mystical feeling among his contemporaries [9, p. 73] ("Pascal's mystic hexagon"). Chasles' theorem evokes the same feeling today.

The relevance of Chasles' theorem has been aided by the development of 3D computer geometric simulation in architectural and construction design and the widespread use of polyhedrons and quadrics as objects of architectural projects.

Chasles' theorem and his references to the works of the 19th century [10] are difficult to understand. There are no illustrations explaining the theorem. Perhaps for these reasons the theorem was forgotten. Over a century and a half of / after its publication, we found only one article [2] and a brief reference to Chasles' theorem [11, p. 99].

[2] gives a proof for Chasles' theorem as exemplified by an ellipsoid. It can probably be extended to other quadrics based on projective transformations. However, such an approach complicates the understanding of the theorem.

Our work constructs visual $3 \mathrm{D}$ models revealing the content of Chasles' theorem and its numerous forms.

Chasles' theorem considered the following relative positions of a tetrahedron and a quadric:

1. each edge of the tetrahedron intersects the quadric twice;

2. all the vertices of the tetrahedron belong to the quadric;

3. all the edges of the tetrahedron are tangent to the quadric;

4. all the faces of the tetrahedron are tangent to the quadric;

5. two tangent planes enclosing the quadric are constructed through each edge;

6. there are also several examples from projective geometry.

In this paper, we consider $1-5$. Within the boundaries of each form, the shape of the tetrahedron and the type and parameters of the quadric can be arbitrary.

When creating models, we used well-known methods of construction in AutoCAD [12], Lisp for constructing a Hyper model using three guides [13], and constructing conics by the five parameters [14]. We took into account the study and application of Pascal's hexagon [15], constructing 3D models of quadrics [16], and using 3D parametrization of SolidWorks [17].

\section{The edges of the tetrahedron twice intersect the quadric (variant 1)}

Each edge of the tetrahedron intersects the surface of the quadric at two points. The edges can intersect the quadric in its real section or on its continuation.

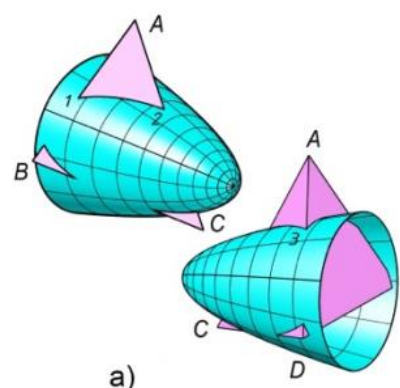

a)

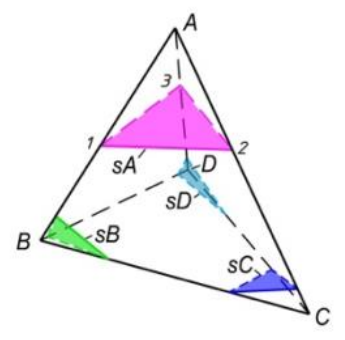

b)

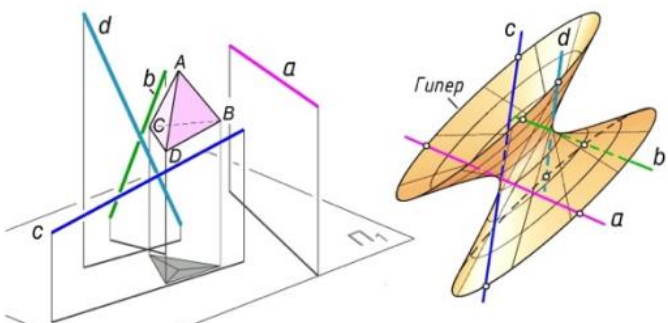

d)

Fig. 1. Intersection of a tetrahedron with a paraboloid: $a-$ model; $b-$ triangular sectors; $c-$ four segments of the guides; $d$-control Hyper 
Let us consider an example of when a tetrahedron $A B C D$ is intersected with a rotational paraboloid (Fig. 1, $a$ ). Each edge intersects the quadric in its real section twice. We find the points where the edges intersect the quadric. We combine these points from the side of each vertex into triangles (Fig. 1, b). Then, we find the intersection of each triangle with the opposite face of the tetrahedron. For example, the triangle $s A(1,2,3)$ is constructed from the side of the vertex $A$. Its intersection with the face $B C D$ opposite the vertex $A$, the straight line $a$ is formed (Fig. 1, c). Four line segments $a, b, c, d$ are found for the four vertices $A, B, C, D$ :

$a=s A \cap(B C D) ; b=s B \cap(A C D) ; c=s C \cap(A B D) ; d=s D \cap(A B C)$.

According to Chasles' theorem, the segments $a, b, c, d$ are the guides of a single Hyper (Fig. 1, d).

\section{Experimental verification of the accuracy of constructions}

We experimentally verify that the four constructed segments $a, b, c, d$ are the guides of the single Hyper. First, we confirm that the line segments belong to the skew lines by checking for the absence of paired intersections of these segments. Then we apply two verification methods.

First, we choose three arbitrary segments of the four, for example, $a, b, c$. We take these segments as guides and, using Lisp [13], construct a surface of the control hyperboloidHyper (Fig. 1, $d$ ). We verify that the fourth segment $d$ also belongs to the surface of this Hyper.

To verify and assess the accuracy of the constructions, we determined the distance from the segment $d$ to the elliptical bases of Hyper or the cross-section constructed by the end points of the segment $d$ and the Hyper center. The distance did not exceed $0.01 \%$ of the typical dimensions of the tetrahedron, which indicates the high accuracy of the constructions and experimentally confirms Chasles' theorem. To illustrate, we marked the intersection points of segments $a-d$ with ellipses by inserting round markers at these points (see Fig. 1, $d$ ).

The second verification method is based on the fact that there are two families of straight lines-guides and generators-on the Hyper surface [3-6]. All the generators intersect all the guides. Therefore, we verify the possibility of constructing a certain segment $m$ intersecting all four segments $a, b, c, d$. If $m$ is found, Chasles' theorem is confirmed.

The segments $a, b, c, d$ were exported from AutoCAD to SolidWorks. Using 3D parameterization, we found the fifth segment $m$ intersecting the first four [17]. It is known that in the general case, there are two segments intersecting four arbitrarily given skew lines [17]. However, in this example, we can move the segment $m$ in space while preserving the intersection with the other four. Consequently, the segment $m$ belongs to the family of the generators of the Hyper, in which the segments $a, b, c, d$ are the guides, which confirms Chasles' theorem.

\section{The proof of Chasles' theorem}

For the first time, a proof of Chasles' theorem was presented in [2]. It is given for the intersection of a tetrahedron with an ellipsoid, in which the intersection points are located in the real part of the edges. We repeated the same proof [18] for an example with a paraboloid (see Fig. 1). 


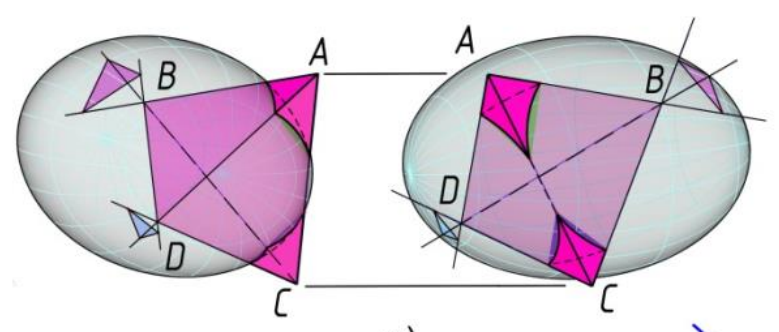

a)

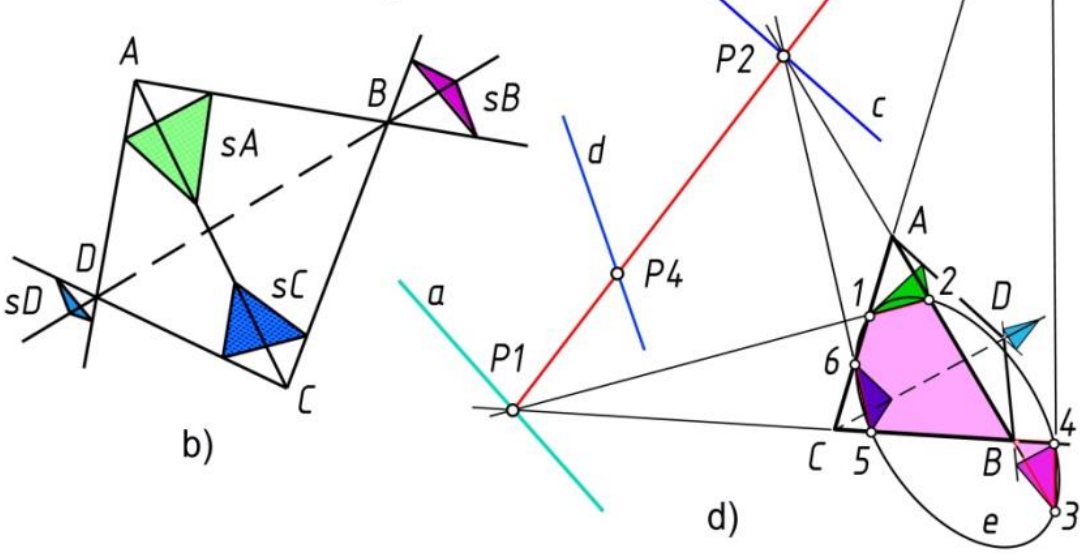

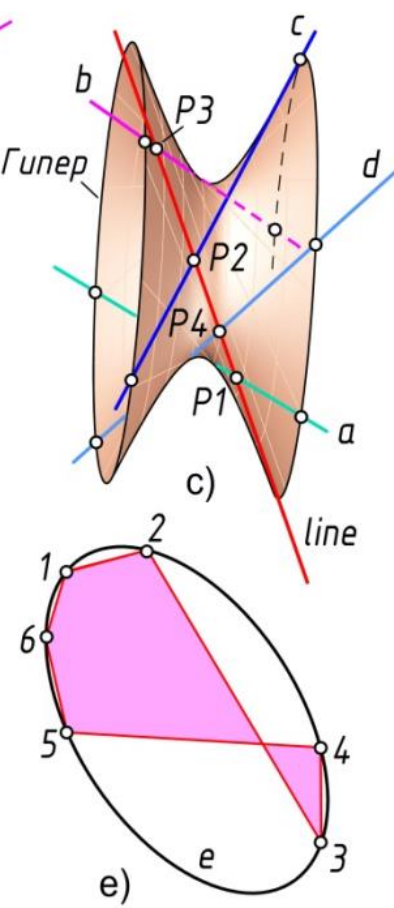

e)

Fig. 2. Intersection of a tetrahedron with an ellipsoid: $a$-model; $b$-triangular sectors; $c-$ control Hyper; $d$-to the proof of the theorem; $e$-Pascal's hexagon

Now we construct a proof for a more general example with a combined arrangement of the tetrahedron vertices. A triaxial ellipsoid was chosen as a quadric (Fig. 2, a). Vertices $A, C$ are outside the quadric, and vertices $B, D$ are inside the quadric.

We find the points of intersection of the edges with the quadric. For the outer vertices $A, C$, these points are located in the real segments of the edges, for the inner vertices $B, D-$ on the continuation of the edges. We combine the points on the side of each vertex into triangles (Fig. 2, b). We find the segments $a, b, c, d$ of the intersection of the triangles with the opposite faces of the tetrahedron. Using three of them, for example, $a, b, c$, we construct the control Hyper (Fig. 2, c) and experimentally verify that the segment $d$ belongs to the Hyper surface.

To prove the theorem, we choose one of the faces of the tetrahedron, for example, $A B C$ (Fig. 2,d). The choice is determined only by the visualization of the subsequent constructions. When the quadric intersects this face we obtain a conic, in our example an ellipse $e$. Connecting points 1-6 of the intersection of the edges of the conic face, we obtain one of Pascal's hexagons (Fig. 2, d). The choice of the point connection order affects only the visualization of the constructions. We find points $P 1=(1-2) \cap(4-5), P 2=(2-3) \cap(5-6)$, $P_{3}=(3-4) \cap(6-1)$. According to Pascal's theorem, points $P_{1}, P_{2}, P_{3}$ belong to a single straight line-Pascal's line-which is easy to verify experimentally.

Since $P_{1} \subset(1-2)$, then $P_{1} \subset s A, P_{1} \subset(4-5)$ and $P_{1} \subset(B C D)$. Consequently, $P_{1}$ belongs to the line of intersection of the triangles $s A$ and $B C D$. However, the line of intersection of these triangles, according to the algorithm of the theorem, is the straight line $a$, therefore $P 1$ belongs to the straight line $a$.

Analogous conclusions for points $P_{2}, P_{3}$ are:

$P 2 \subset(5-6), P 2 \subset s C ; P 2 \subset(2-3), P 2 \subset(A B D) \Rightarrow P 2 \subset c(c=s C \cap A B D)$;

$P_{3} \subset(3-4) P_{3} \subset s B ; P_{3} \subset(6-1), P_{3} \subset(A C D) \Rightarrow P_{3} \subset b(b=s B \cap A C D)$.

Since the points $P_{1}, P_{2}, P_{3}$ belong to the line, the line intersects the lines $a, b, c$.

The straight line $d$ is obtained at the intersection of the $A B C$ plane with the triangle $s D$. Consequently, $d \subset A B C$. The line is also in the $A B C$ plane by its construction. Therefore, the lines $d$ and line intersect. Their intersection point is designated $P 4$. 
The line intersects all the lines $a, b, c, d$. Hence, the lines $a-d$ are the guides of a single Hyper (see the second verification method above). The theorem is proved.

Since the line intersects the Hyper guides, it belongs to its generatrix family. This was also confirmed by the Hyper model, which experimentally verifies that the line belongs to its surface (see Fig. 2, c).

If, instead of the ellipsoid, we chose another quadric, the line of reasoning would be the same. The constructions lead to another conic, including a hyperbola or a parabola. According to Pascal's theorem, the points $P_{1}, P_{2}, P_{3}$ belong to the single straight line. The point $P_{4}$ also belongs to this line, which leads to a proof of Chasles' theorem.

\section{Additional examples of variant 1}

We constructed models for all types of quadrics. All the examples confirmed Chasles' theorem.

For a hyperbolic paraboloid (HypAr) (Fig. 3, a) and a two-sheeted hyperboloid (Fig. 3, c), some of the edges intersect the quadric in the real section, and some on the continuation (combined variant). For a one-sheeted hyperboloid (Fig. 3, b) and a cylinder (Fig. 3, d), all the edges intersect in the real section.

We obtained other models with numerous variants of the intersection of the edges of a tetrahedron with a quadric. The exception is the HypAr, for which we managed to construct a model only with a combined intersection variant.

The model for a two-sheeted hyperboloid is realized both with the intersection of two bowls (Fig. 3, c), and with one bowl, similar to a paraboloid (see Fig. 1, a).

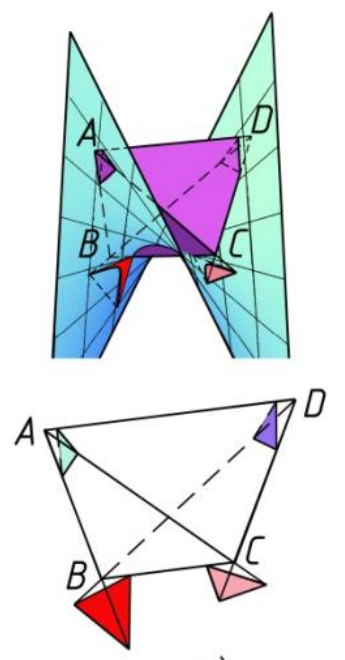

a)
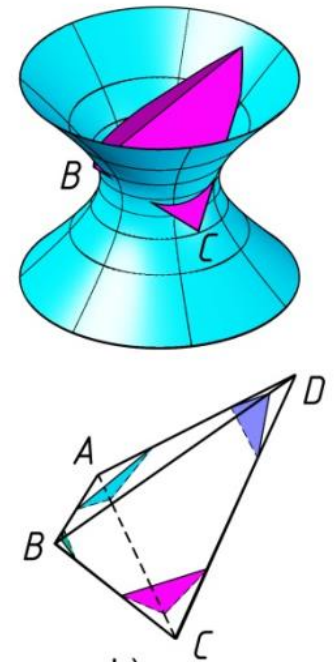

b)

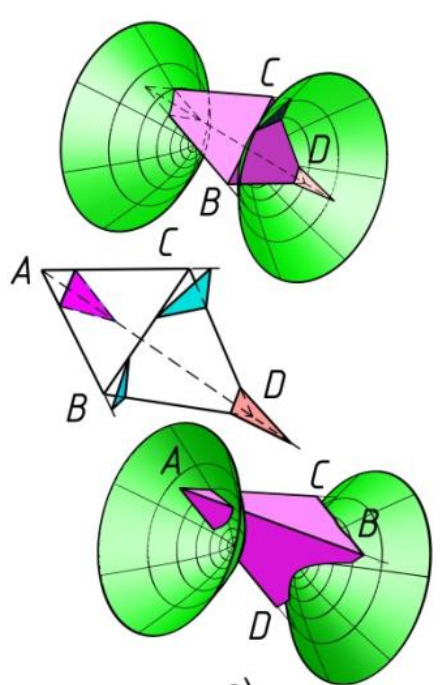

c)
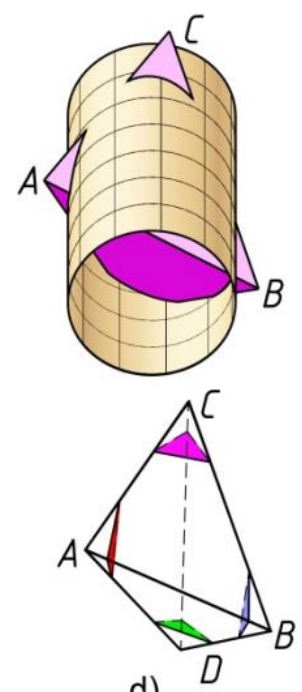

d)

Fig. 3. Intersection of a tetrahedron with different quadrics: $a$ - hyperbolic paraboloid; $b-$ one-sheeted hyperboloid; $c$ - two-sheeted hyperboloid; $d$ - cylinder

\section{All the vertices of the tetrahedron belong to the quadric (variant 2)}

Chasles considered this variant as a limiting process when the vertices of the tetrahedron approach the surface of the quadric, during which the triangular sections are transformed into the tangent planes to the quadric. Taking into account this assumption, the above proof can be applied to this variant. 


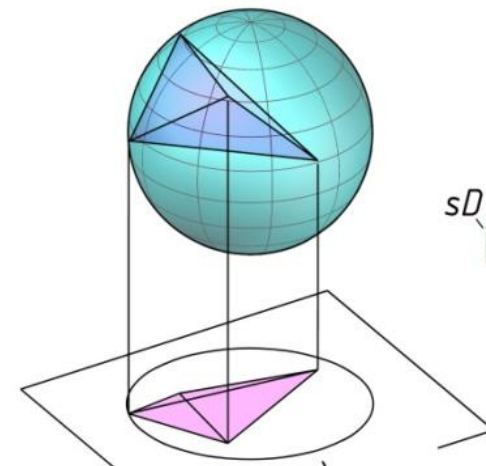

a)

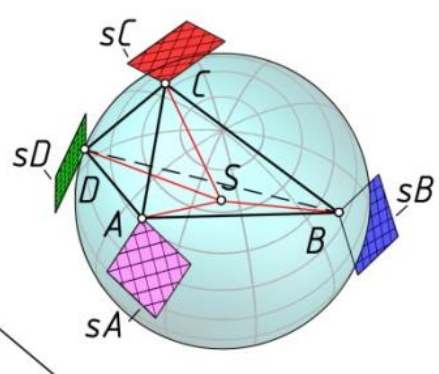

b)

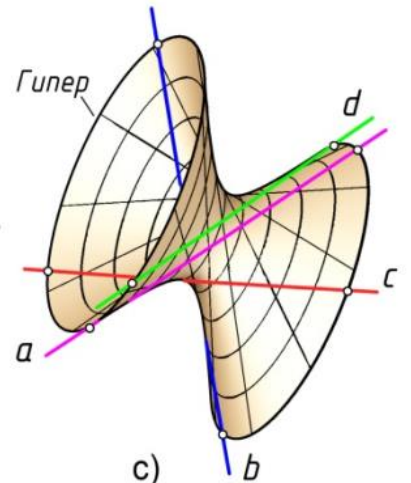

c)

Fig. 4. The tetrahedron is inscribed in the sphere: $a$-model; $b$-tangent planes; $c$-guides of the control Hyper

Let us consider an example when the vertices of the tetrahedron $A B C D$ are located on a sphere (Fig. 4, a). At the vertices, we construct tangent planes perpendicular to the segments connecting the vertices with the center of the sphere $S$. These planes are shown as sectors $s A-s D$ (Fig. $4, b$ ). We construct line segments of the intersection of the tangent planes with the tetrahedron faces opposite the vertices. For example, the segment $a=s A \cap$ (BCD). We choose three arbitrary segments from $a, b, c, d$ and use them to construct a Hyper (Fig. 4, c). We verify that the fourth segment also belongs to the Hyper surface, which confirms Chasles' theorem.

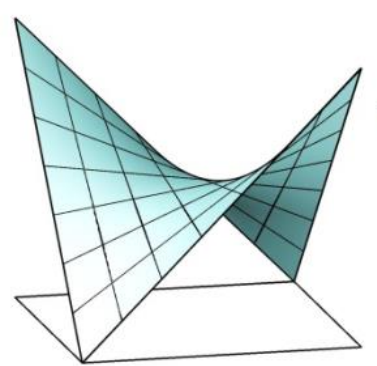

a)

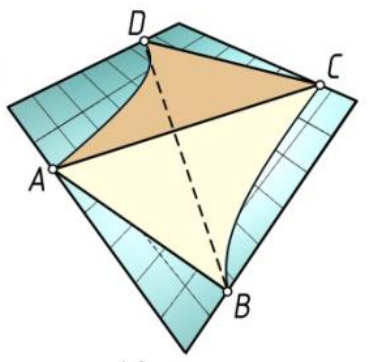

b)

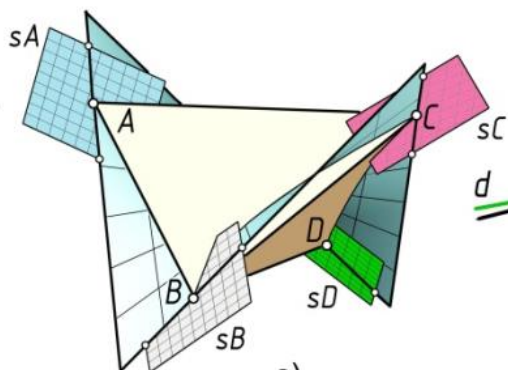

c)

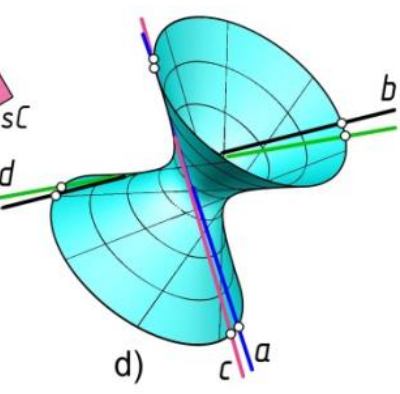

d)

Fig. 5. The tetrahedron is inscribed in a hyperbolic paraboloid (HypAr): $a, b-$ model; $c-$ tangent planes; $d$-guides of the control Hyper

The second example is when the vertices of the tetrahedron are located on the $H y p A r$ surface (Fig. 5, a). The vertices of the tetrahedron (Fig. 5, b) and the tangent planes at the vertices (Fig. 5, c) are set by the lined frame of the HypAr. The construction of the control Hyper (Fig. 5, d) in this example also confirms Chasles' theorem.

Control Hypers with narrow elliptical necks were obtained in the models (Fig. 4, Fig. 5). Such Hypers also appeared in other experiments. For illustration, the Hypers together with the guide segments were scaled, setting different scales along the axes.

\section{The edges of the tetrahedron are tangent to the quadric (variant 3)}

A tetrahedron whose edges are tangent to the quadric is called framed. A framed tetrahedron $(F T)$ can be constructed only for convex quadrics (sphere, ellipsoid, paraboloid, two-sheeted hyperboloid). Therefore, this version of the theorem has limited manifestations. 


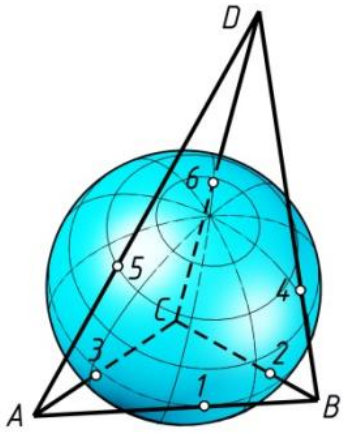

a)

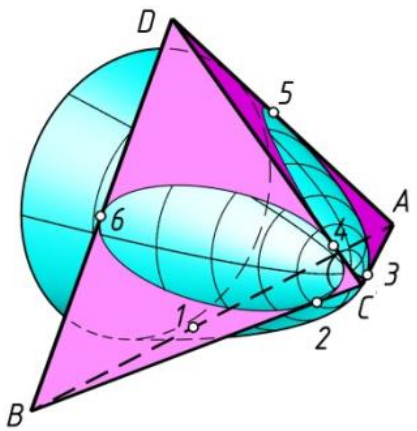

b)

Fig. 6. Framed tetrahedrons: $a$-for a sphere; $b$-for a paraboloid

We construct the FT for a sphere based on its properties [19]: the sums of the lengths of the skew edges are equal between themselves. For the tetrahedron $A B C D$ (Fig. 6, $a$ ), we write: $A B+C D=A C+B D=A D+B C=S$. For example, taking $S=140$, we obtain the values $A B=70 ; B C=50 ; A C=60 ; A D=90 ; C D=70 ; B D=80$. We construct the face $A B C$. We find the vertex $D$ at the intersection of three auxiliary spheres with their centers at the vertices $A, B$, $C$ and the radiuses $A D, B D, C D$, respectively. We plot inscribed circles on two faces. We construct perpendiculars from the centers of these circles and we find the center of the inscribed sphere at their intersection [20]. Then we determine the radius of the inscribed sphere and six points of tangency $1-6$.

For the remaining quadrics: the ellipsoid, the paraboloid (Fig. 6, b) and the two-sheeted hyperboloid (Fig. 7, a), this property of the $F T$ sides is not satisfied. Therefore, the $F T$ was constructed in SolidWorks using 3D parameterization. We constructed the surface of the quadric and six segments tangent to the quadric. The vertices of the segments were united. To set the tangent planes at the points of tangency, we constructed line segments perpendicular to the quadric.

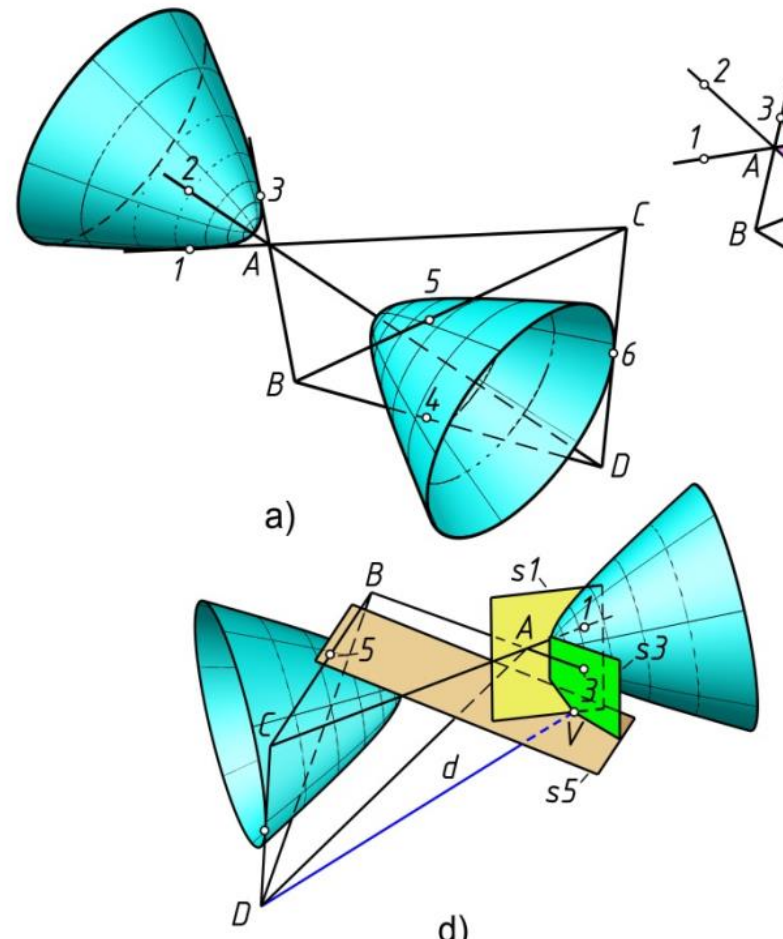

d)
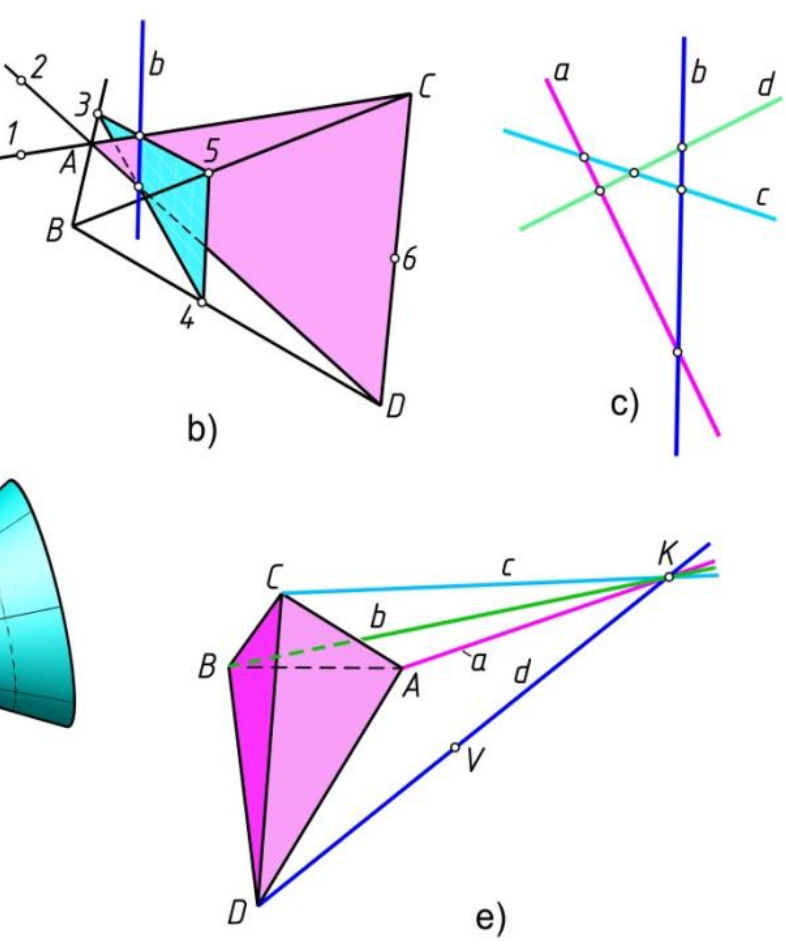

Fig. 7. The edges of the tetrahedron are tangent to the two-sheeted hyperboloid: $a-$ model;

$b$-construction of the segment $b$ for the vertex $B ; c-$ four coplanar segments; $d-$ construction of the segment $d$ for the face $A B C ; e$-four intersecting segments 
In Chasles' theorem, there are two clauses for framed tetrahedrons-clause 3 and clause 7. Various construction algorithms are provided for these points. Let us consider them as exemplified by a two-sheeted hyperboloid (Fig. 7).

According to clause 3, the points of tangency belonging to the edges going from one vertex are combined into triangular sectors. There are four such sectors (by the number of vertices). We find the lines of intersection of each sector with the face opposite the vertex of this sector. We repeat this operation for each vertex. For example, for the vertex $B$ we construct a sector (3-4-5) and a segment $b=(3-4-5) \cap(A C D)$ (Fig. $7, b)$. As a result, we obtain segments $a, b, c, d$ for all the vertices (Fig. $7, c$ ). The experiment shows that these segments are coplanar.

According to clause 7 , tangent planes to the quadric are constructed at the points of tangency belonging to the edges of a single face. There are three such planes for each face (by the number of edges of the triangular face). We consider further constructions on the example of the face $A B C$ (Fig. 7, d). The points of tangency of the edges of this face are 1, 3, 5 . The tangent planes to the quadric at these points are shown by the rectangles $s 1, s 3, s 5$. We find the vertex $V$ of the trihedral angle formed by these tangent planes. We connect this vertex by the segment $d$ with the vertex $D$ of the tetrahedron opposite the considered face. We repeat the construction for each face and obtain four segments $a, b, c, d$ (Fig. $7, e$ ). These constructions show that these segments intersect at one point $K$, wherein, there are no coplanar triples of segments among them.

Thus, for $F T$, we obtain either four coplanar segments or four non-coplanar segments with a common intersection point. Our result differs from the conclusions of Chasles, who believed that the four segments belong to the surface of a single hyperboloid. However, he pointed to an analogy with Brianchon's theorem [1, 7], in which the diagonals of a hexagon circumscribed around a conic intersect at one point.

\section{The faces of the tetrahedron are tangent to the quadric (variant 4)}

Let us consider the construction of a tetrahedron, whose faces are tangent to a quadric, using an example with a two-sheeted hyperboloid. We set four points $A, B, C, D$ on the surface of the hyperboloid (Fig. 8). The positions of the points are determined only by the condition of the construction visualization. We construct tangent planes at these points. Each plane is set by two straight lines tangent to the quadric at the selected point. For construction visualization, the tangent planes are displayed by the rectangles $s A-s D$.

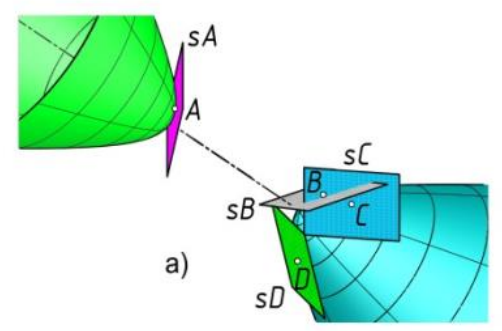

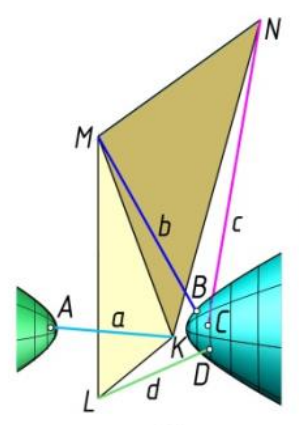

b)

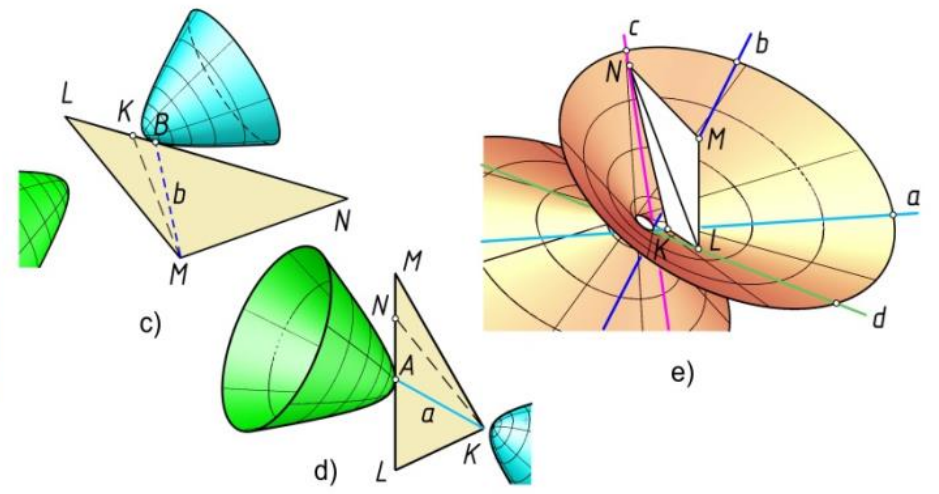

Fig. 8. The tetrahedron faces are tangent to the two-sheeted hyperboloid: $a$-tangent planes; $b-$ a tetrahedron and guide segments; $c$-tangency at the vertices; $d$-control Hyper

For three planes, for example, $s B, s C, s D$, we find the vertex of the trihedral angle-point $K$. We cut the triangular angle with the fourth plane $s A$. Using the triangle of the section $L M N$ 
and the vertex $K$, we construct a tetrahedron $K L M N$, all the faces of which are tangent to the quadric. In the given example (Fig. 8, b) the tangency is achieved on the continuation of the faces.

According to the algorithm of Chasles' theorem, we connect the points of tangency of the faces with the vertices of the tetrahedron opposite the faces (Fig. 8, b). In our example (Fig. $8, c, d)$, the face $L M N$ is tangent to the hyperboloid at point $A$. The opposite vertex is $K$ for this face. Therefore, the segment is $a=A K$. The face $K L N$ has a point of tangency $B$ and its opposite vertex $M$. Therefore, the segment is $b=B M$, etc.

We arbitrarily choose three of the four segments, and use them to construct a control Hyper (Fig. 8,e). The fourth segment also belongs to the surface of this Hyper, which confirms Chasles' theorem.

Since the segments $a-d$ pass through the vertices of the tetrahedron, these vertices belong to the surface of the control Hyper (see Fig. 8, e).

\section{The tangent planes from the edges of the tetrahedron enclose the quadric (variant 5)}

According to this variant of the theorem, there should be 12 tangent planes enclosing the quadric, i.e., two planes for each edge of the tetrahedron. Hence, the edges of the tetrahedron should not be tangent to the quadric or intersect it.

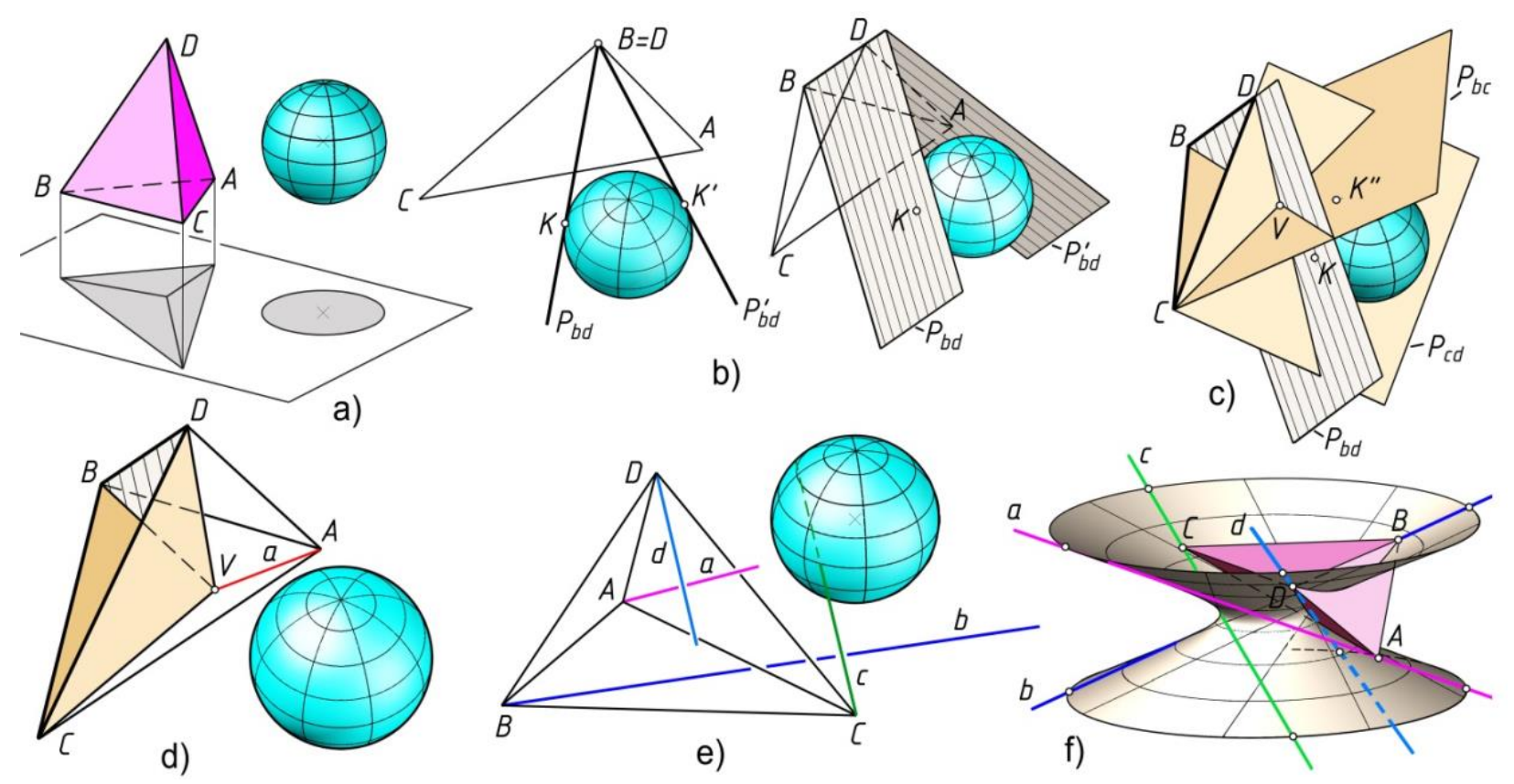

Fig. 9. Tangent planes from the edges of the tetrahedron to the sphere: $a$-model; $b-$ tangent planes for the edge $B D ; c$-triangular angle for the face $B C D ; d$-one of the segments of the guides; $e$-one of the variants of four guides; $f$-control Hyper

We initially studied this variant using the example of a sphere. The sphere could be located either outside the tetrahedron (Fig. 9, $a$ ) or be completely or partially embedded in the tetrahedron.

Let us consider the face $B C D$ of the tetrahedron $A B C D$. For the edge $B D$ (Fig. $9, b$ ), there are planes $P_{B D}$ and $P_{B D}{ }^{\prime}$ tangent to the sphere, which touch it at points $K$ and $K^{\prime}$. There are six tangent planes for the selected face. Eight triangular angles can be built from them. This is determined by the number of combinations of three edges, two tangent planes for each edge, i.e. $2^{3}$.

Let one of the trihedral angles of the face $B C D$ (Fig. 9, $c$ ) have a vertex $V$. According to the algorithm of the theorem, this vertex should be connected by a segment with the vertex of 
the tetrahedron opposite the face. The vertex of the tetrahedron opposite the face $B C D$ is the vertex $A$. We construct the segment $d=A V$ (Fig. 9, d). Eight variants for the segment $d$ are possible for the eight triangular angles of the face $B C D$.

After similar constructions, we find segments for each face. Let us denote them by the name of the tetrahedron vertices, to which they are directed, i.e., $a, b, c, d$. Each of these segments has eight construction variants (according to the number of vertices of the triangular angle for each face).

Thus, the problem of constructing the segments connecting the vertices of the trihedral angles with the vertices of the tetrahedron is multivariate. Choosing one segment of each face out of the eight possible ones, we obtain $84=4096$ combinations of the segments $a-d$ for the four faces, i.e., 4096 construction variants. This is the number of combinations of 4 elements (faces) with 8 variants of each element (segments of each face).

We arbitrarily selected the tangent planes. For example, a plane could be selected twice as belonging to two faces with a common edge. Or a plane could be missed. However, for such a choice, the result was most often negative, i.e., the theorem was not confirmed.

Since the theorem does not say how to choose the tangent planes, we assumed that the reason for the negative results is connected with the incorrect choice of the tangent planes. To determine the conditions for the realization of the theorem, we developed a Lisp program, which analyzed all possible combinations of planes and identified those in which the theorem was fulfilled. This revealed that out of the 4096 variants, only 64 led to the realization of the theorem. Their analysis allowed us to formulate the following necessary and sufficient condition for the realization of the theorem: "Each of the twelve tangent planes should participate in the construction of the model once and only once". The resulting value coincides with the number of combinations of six elements (edges), two variants (planes) for each element, i.e. $2^{6}=64$.

Let us consider one of the 64 variants, in which we obtained the segments $a-d$ (Fig. 9, $e$ ). We build the control Hyper for three of them, for example, a, b, c (Fig. 9, $f$ ). The verification (see Section 3) showed that the fourth segment $d$ also belongs to the surface of this Hyper, which confirms Chasles' theorem.

This algorithm was experimentally verified for all types of quadrics, with the exception of the cylinder and the cone, for which there are no tetrahedrons with tangent planes from all the edges to the quadric.

The experiments with quadrics were carried out as follows. A visual surface of the quadric was set. Then, the parameters and position of the tetrahedron were experimentally selected so that it would be possible to construct two tangent planes to the quadric surface from each of its edges. The parameters of the 12 tangent planes were entered into the Lisp program, which checked 4096 possible construction variants and found 64 variants satisfying the theorem. We established that, as in the example with the sphere, the 64 variants are realized only when the above condition on the combination of tangent planes is met. Let us consider some examples.

For a rotational paraboloid and a tetrahedron $A B C D$ (Fig. 10, $a$ ), the edge $A B$ and the paraboloid were projected onto the plane $\Sigma \perp A B$. From the point $A^{\prime}=B^{\prime}$, we found tangent lines $m, n$ to the outline of the paraboloid projection-the parabola $p$ (Fig. 10, b). We found the tangents $m^{\prime}, n^{\prime}$ for the edge $A D$ (Fig. 10,c). We also found the tangents for the remaining edges of the tetrahedron. The edges and the corresponding tangent lines formed the desired tangent planes. We applied the program to the 12 tangent planes, which revealed the 64 variants with a positive solution. For the most illustrative variant, we constructed a control Hyper (Fig. 10, $d$ ) with the segments $a, b, c, d$ and confirmed that these segments belong to the Hyper surface. 


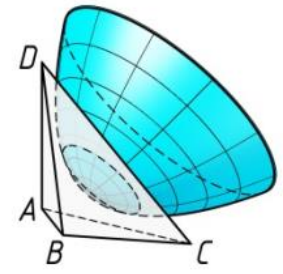

a)

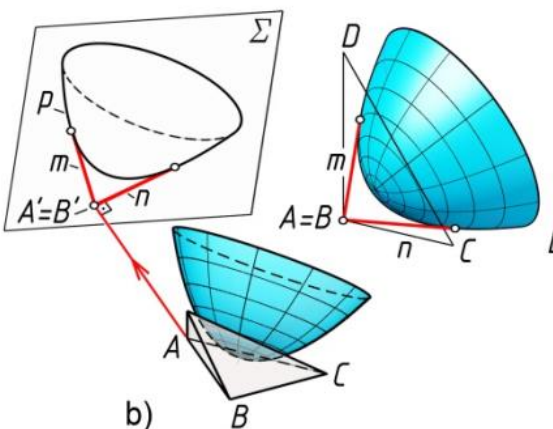

b)

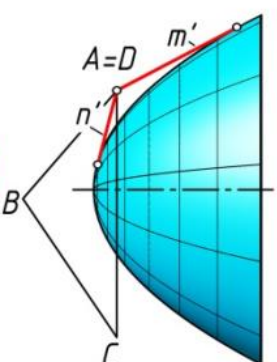

c)

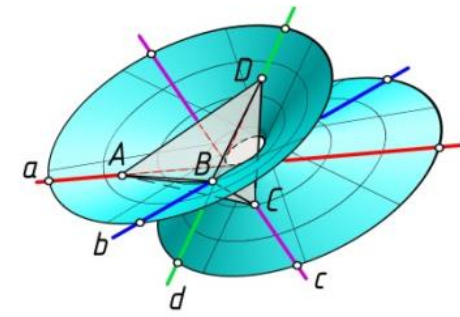

d)

Fig. 10. Tangent planes from the edges of the tetrahedron to the paraboloid: $a-$ model; $b-$ tangent planes for the edge $A B$; $c$-tangent planes for the edge $A D ; d$-segments of guides and control Hyper

For a one-sheeted hyperboloid, we constructed the tetrahedron $A B C D$ (Fig. 11, $a$ ). Using a projection onto the plane perpendicular to the edges, we found the tangent planes from all the edges to the hyperboloid surface, for example, for the edge $A D$ (Fig. 11, $b$ ) and the edge $A B$ (Fig. 11, c). We found segments $a, b c d$. The construction of the control Hyper confirmed that these segments belong to its surface (Fig. 11, $d$ ).

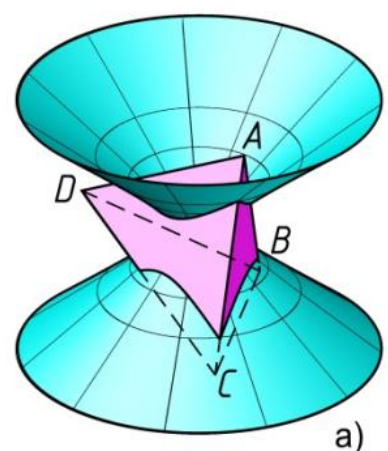

a)

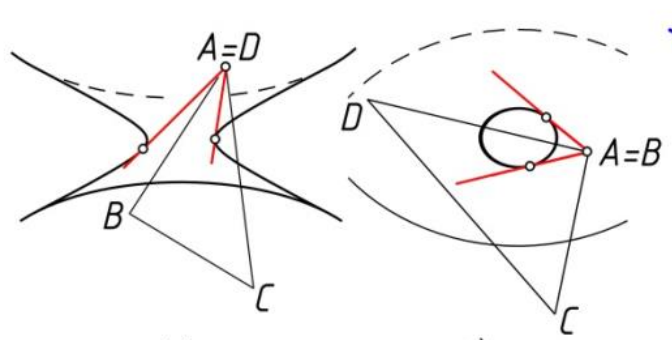

b)

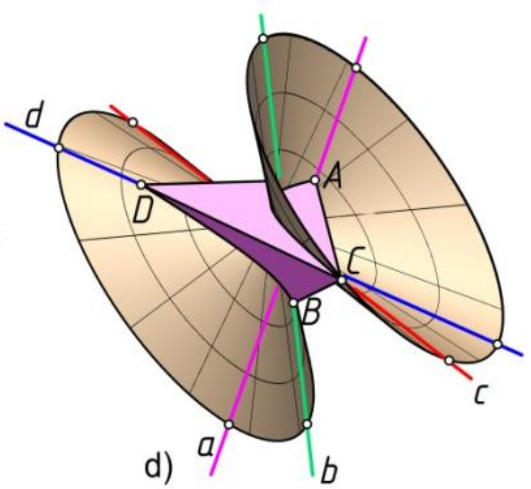

Fig. 11. Tangent planes from the edges of the tetrahedron to the one-sheeted hyperboloid: $a$-model; $b$-tangent plane of the edge $A D ; c$-tangent plane of the edge $A B ; d-$ segments of guides and control Hyper

Since the segments of the guides $a, b c d$ are constructed from the vertices of the tetrahedron, these vertices belong to the surface of the control Hyper (see Fig. 9, $f$; 10, $d$; $11, d)$.

Let us note one more feature of this variant of the theorem. The control Hypers obtained for the 64 variants of the single model intersect pairwise when the fourth-order intersection line splits into straight lines and conics. For example, in the experiments with a sphere (see Fig. 9), we obtained control Hypers, the intersection lines of which were two intersecting straight lines and an ellipse (Fig. 12, a), two intersecting straight lines and a hyperbola (Fig. 12, b), and four pairwise intersecting straight lines (Fig. 12, d). 


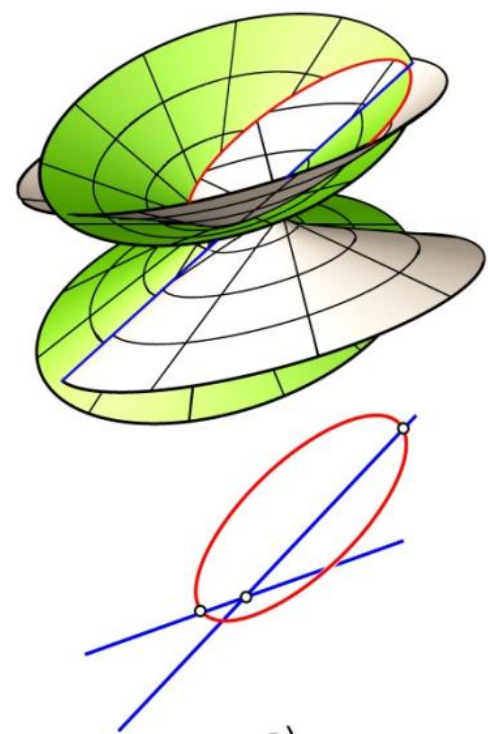

a)
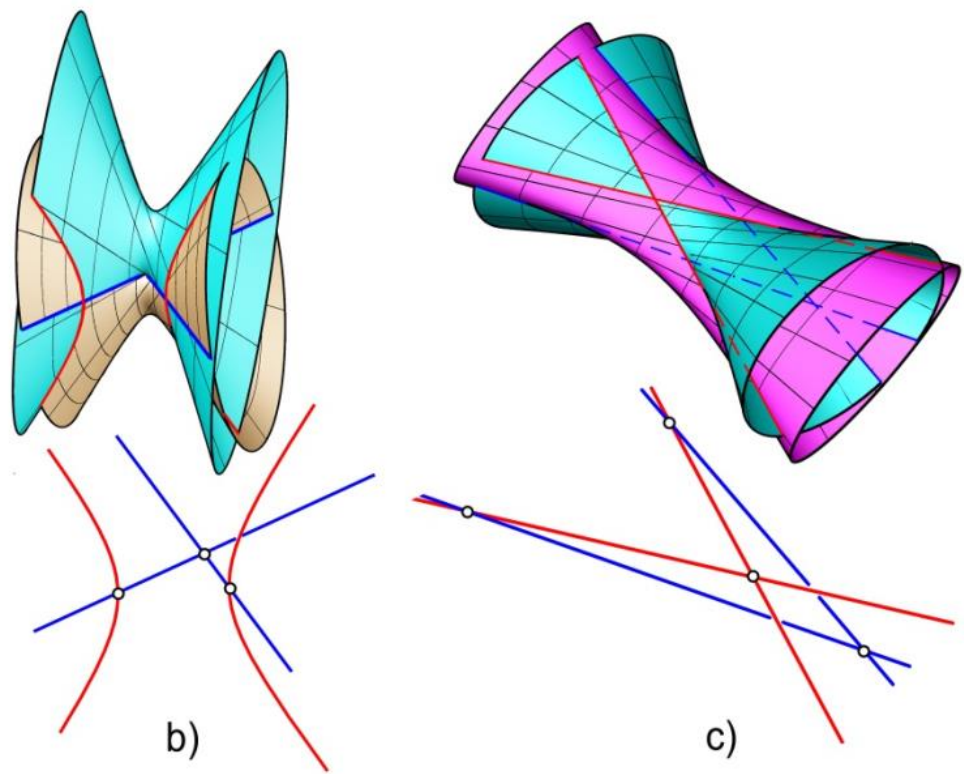

Fig. 12. Examples of the mutual intersection of control Hypers: $a$-two intersecting straight lines and an ellipse; $b$-two intersecting straight lines and a hyperbola; $c$-four pairwise intersecting straight lines

\section{Conclusion}

We obtained visual geometrically accurate [15] models for all the variants of Chasles' theorem. The models required complex $3 \mathrm{D}$ geometric constructions.

The models confirmed the conclusions of Chasles' theorem as applied to its main variants. However, for the framed tetrahedron, we obtained a conclusion different from Chasles.

The proof of Chasles' theorem was found only for the first and second variants of the theorem. The problem of finding proofs for all the variants or to find a universal proof of the theorem remains.

The analogy between the theorems of Chasles and Pascal allows us to assume that Chasles' theorem could have the same essence in geometric simulation as Pascal's theorem.

The models above are included as relevant problems in a new course on the theoretical foundations of $3 \mathrm{D}$ computer geometric simulation. The course is meant for students of engineering specialties as an alternative to descriptive geometry [21].

Our article is among the works showing the current interest in historical works on geometry [22], including those of Chasles [23]. It also demonstrates new possibilities for studying historical and modern problems [24] based on computer 3D geometric modeling.

\section{Acknowledgements}

The author is grateful to Alexandr V. Seliverstov for advice on the topic of this article.

\section{References}

1. Chasles M. Istoricheskij obzor proisxozhdeniya i razvitiya geometricheskix metodov. Istoriya geometrii. Primechaniya [Historical review of the origin and development of geometric methods. History of geometry]. Moscow, V.2, Note XXII. 1883. 428 p. [in Russian]

2. Morducai-Boltovsky D. D. Tryoxmerny `j i chety ‘ryoxmerny ` $j$ analogon teoremy ` Paskalya [Three-dimensional and four-dimensional analog of Pascal's theorem] / Advances in mathematical Sciences. Vol. VIII, № 2 (54). 1953, pp. 135-138. [in Russian] http://mi.mathnet.ru/umn8192

3. Peklich V. A. Nachertatel naya geometriya [Descriptive geometry]. Moscow, 2007, ASV, 272 p. [in Russian] 
4. Chetverukhin N. F. Nachertatel 'naya geometriya [Descriptive geometry]. Moscow, 1963, 420 p. [in Russian]

5. Ivanov G. S. Nachertatel 'naya geometriya [Descriptive geometry]. Moscow, 1995, 224 p. [in Russian]

6. Ivanov G. S. Teoreticheskie osnovy' nachertatel 'noj geometrii [Theoretical Foundations of Descriptive Geometry]. Moscow, 1998, 158 p. [in Russian]

7. Chetverukhin N. F. Proektivnaya geometriya [Projective geometry]. Moscow, 1961, 360 p. [in Russian]

8. Glagolev N. A. Proektivnaya geometriya [Projective geometry]. Moscow, 1963, 344 p. [in Russian]

9. Chasles M. Istoricheskij obzor proisxozhdeniya i razvitiya geometricheskix metodov. Istoriya geometrii [Historical review of the origin and development of geometric methods. History of geometry]. Moscow, V.1., 1883, 311 p. [in Russian]

10. Chasles. Géométrie de situation. Démonstration de quelques propriétés du triangle, de l'angle trièdre et du tétraèdre, considérés par rapport aux lignes et surfaces du second ordre / Annales de mathématiques pures et appliquées. V.19, 1828-1829, pp. 65-85. http://www.numdam.org/volume/AMPA_1828-1829__19/

11. Prasolov V. V., Tikhomirov V. M. Geometriya [Geometry]. Moscow, 2007, 328 p. [in Russian]

12. Kheifetc A. L., Loginovskiy A. N., Butorina I. V., Vasileva V. N. Inzhenernaya 3Dkompyuternaya grafika [Engineering 3D computer graphics]. Moscow, "Yurayt", 2015, 602 p. [in Russian]

13. Kheyfets A. L., Loginovsky A. N. 3D-modeli linejchaty'x poverxnostej s tremya pryamolinejny 'mi napravlyayushhimi [3d-models of ruled surfaces with three rectilinear guides] Cheljabinsk: Bulletin of the South Ural State University Series "Construction Engineering and Architecture". V. 7, 2008, № 25(125), pp. 51-56. [in Russian]

http://dspace.susu.ru/xmlui/bitstream/handle/ooo1.74/615/10.pdf?sequence=1\&isAl lowed $=\mathrm{y}$

14. Korotkii V. A., Kheyfets A. L. 3D-modelirovanie konik $v$ pakete AutoCAD [3D modeling of conics in AutoCAD]. Actual issues of graphic education of youth. Materials of the VI All-Russian scientific-methodical conference, Rybinsk: RGTA, 2005, pp. 102-105. [in Russian]

15. Kheyfets A. L. Geometricheskaya tochnost` komp 'yuterny ‘x algoritmov konstruktivny'x zadach [Geometrical accuracy of computer algorithms for constructive problems]. The problem of the quality of the graphic preparation of students in technical high school: tradition and innovation, V. 3, Perm: PNRPU, 2016, pp. 367-87. [in Russian] http://dgng.pstu.ru/conf2016/papers/74/

16. Kheyfets A. L. 3D-model of ruled surface with three curvilinear guides / 11 th International Conference 3IA'2008, the International Conference in Computer Graphics and Artificial Intelligence. 30-31 May 2008. Athens. Greece Eurographics. Organised by: XLIM Laboratory University of Limoges. 2008, pp. 223-227. [in Russian]

17. Kheyfets A. L. $3 D$ modeli $i$ algoritmy 'komp `yuternoj parametrizacii pri reshenii zadach konstruktivnoj geometrii (na nekotory ' $x$ istoricheskix primerax) [3D Models and Algorithms for Computer-Based Parameterization for the Decision of Tasks of Constructive Geometry (at Some Historical Examples)]. "Bulletin of the South Ural State University", Series "Computer Technologies, Automatic Control, Radioelectronics". Cheljabinsk, V. 16. №2, c. 24-42. [in Russian] https://vestnik.susu.ru/ctcr/article/view/4909/4293

18. A. L. Kheifetc Kheyfets, "Chasles' Theorem as a 3D Analogue of Pascal's Theorem," $2020 \mathrm{~V}$ International Conference on Information Technologies in Engineering 
Education (Inforino), Moscow, Russia, 2020, pp. 1-5, doi: 10.1109/Inforino48376.2020.911169o. https://ieeexplore.ieee.org/document/9111690

19. Karkasny $j$ tetrae $~ d r \quad$ [Wireframe tetrahedron] https://studbooks.net/2395470/matematika_himiya_fizika/karkasnye_tetraedry

20. Poluvpisannaya [Half sfera inscribed sphere]. http://www.myshared.ru/slide/546522/

21. Kheifetc A. L. Nachertatel naya geometriya kak "beg v meshkax". Descriptive geometry as a factor limiting the development of geometric modeling. Proceedings of the V International scientific and practical Internet-conference. Perm: PGTU, 2015, pp. 292-325. [in Russian] http://dgng.pstu.ru/conf2015/papers/72/ http://dgng.pstu.ru/conf2015/news/44/

22. Del Centina A. Pascal's mystic hexagram, and a conjectural restoration of his lost treatise on conic sections // Archive for History of Exact Sciences. 2020. V. 74. P. 469521

https://doi.org/10.1007/s00407-020-00251-2

23. Bussotti P. Michel Chasles' foundational programme for geometry until the publication of his Aperçu historique // Archive for History of Exact Sciences. 2019. V. 73. P. 261308

https://doi.org/10.1007/s00407-019-00222-2

24. Bozoki S, Tsung-Lin Lee, Rónyai L. Seven mutually touching infinite cylinders // Computational Geometry. V. 48, Issue 2, February 2015, pp. 87-93. https://doi.org/10.1016/j.comgeo.2014.08.007 https://habr.com/ru/post/218403/ 